\title{
Prognostic role of telomerase activity in gastric adenocarcinoma: A meta-analysis
}

\author{
MU-HAN LÜ ${ }^{1}$, JIA-QI DENG ${ }^{3}$, YA-LING CAO ${ }^{1}$, DIAN-CHUN FANG $^{1}$, YAO ZHANG ${ }^{2}$ and SHI-MING YANG ${ }^{1}$ \\ ${ }^{1}$ Institute of Gastroenterology, Southwest Hospital, Third Military Medical University; ${ }^{2}$ Department of Epidemiology, \\ Third Military Medical University, Chongqing 400038; ${ }^{3}$ School of Foreign Languages, \\ Lu Zhou Medical College, Luzhou 646000, P.R. China
}

Received November 2, 2011; Accepted January 13, 2012

DOI: $10.3892 /$ etm.2012.471

\begin{abstract}
Activation of telomerase is involved in carcinogenesis in most types of cancers. However, the prognostic value of telomerase activity (TA) in patients with gastric carcinoma (GC) remains controversial. We conducted a meta-analysis to assess the relationship between TA and the clinical outcome of GC. A meta-analysis of 18 studies ( 886 patients) was performed to evaluate the association between TA and metastasis-related parameters in GC patients by searching databases, including PubMed, MEDLINE, EMBASE, Web of Science databases, Cochrane Library and the Chinese Biomedical Literature database (CBM) (last search updated in October 2011). We used the odds ratios (ORs) with $95 \%$ confidence intervals (CIs) to assess the strength of the association between TA and metastasis of GC. Our analysis results indicated that high telomerase activity expression tended to be associated with the presence of lymph node metastasis (866 patients) $(\mathrm{OR}=2.03$, $95 \%$ CI 1.21-3.39, $\mathrm{p}=0.007)$, the depth of invasion (886 patients) $(\mathrm{OR}=1.87,95 \%$ CI 1.30-2.70, $\mathrm{p}=0.0007)$, distant metastasis (407 patients) (OR=2.71, 95\% CI 1.59-4.63, $\mathrm{p}=0.0002)$, tumor size (466 patients) $(\mathrm{OR}=2.14,95 \%$ CI $1.31-3.50, \mathrm{p}=0.002)$ and TNM stage (711 patients) $(\mathrm{OR}=2.39,95 \% \mathrm{CI} 1.30-4.41$, $\mathrm{p}=0.005)$. However, high TA expression was not associated with the presence of histologic differentiation (791 patients) $(\mathrm{OR}=1.51,95 \% \mathrm{CI} 0.73-3.11, \mathrm{p}=0.26)$. In conclusion, telomerase overexpression not only plays a key role in primary initiation, but also promotes invasion and metastatic progression of GC.
\end{abstract}

Correspondence to: Dr Shi-Ming Yang, Institute of Gastroenterology, Third Military Medical University, Chongqing 400038, P.R. China E-mail: shimingyang@yahoo.com

Dr Yao Zhang, Department of Epidemiology, Third Military Medical University, Chongqing 400038, P.R. China

E-mail: sydzy2003@yahoo.com.cn

Abbreviations: hTERT, human telomerase reverse transcriptase; GC, gastric carcinoma; TA, telomerase activity; OR, odds ratio; CI, confidence interval

Key words: telomerase, gastric carcinoma, prognosis, metastasis, meta-analysis
These findings raise the possibility of using TA to screen for the prognosis of gastric cancer.

\section{Introduction}

Gastric carcinoma (GC) is estimated to be the second most common cause of cancer-related death in the world, although both the incidence and mortality have declined in the past 50 years (1). The prognosis of patients with GC remains poor due to the high rate of tumor invasion into underlying tissue and lymph node metastasis, which are major prognostic indicators of neoplastic recurrence after treatment (2). Thus, there is an urgent need to identify cancer metastasis earlier and more accurately. Accumulating evidence indicates that progression beyond the initial stages of the malignant transformation in gastric adenocarcinoma is associated with cellular immortality, which occurs in other neoplasms (3).

The key factor responsible for cellular immortality is telomerase, which is a specialized ribonucleoprotein complex that adds telomeric DNA onto the ends of chromosomes. By synthesizing the repetitive telomeric sequence using its RNA template, telomerase prevents cellular senescence in somatic cells (4). Moreover, human telomerase reverse transcriptase (hTERT), as the rate-limiting step in the activation of telomerase, is known to be an accurate measure of telomerase activity (TA). The presence of hTERT is therefore required for aberrant cell proliferation and carcinogenesis in most cancer types (5). Thus, telomerase is considered to be a potential marker of oncogenesis (6).

The prognostic role of high TA and overexpression of hTERT has been reported by many authors (7,8). Although several studies focusing on telomerase have referred to clinicopathological variables, including tumor size, site, histologic grade, depth of tumor invasion, lymph node metastasis, distant metastasis and TNM stage, the relationship between telomerase and tumor progression or metastasis in patients with GC remains controversial. It is uncertain whether reported results depend on the number of patients or ethnic heterogeneity present in each trial. Therefore, it is appropriate to undertake a meta-analysis of existing trials to achieve insight into the metastatic value of TA and hTERT in GC.

In the present study, we enrolled clinicopathological parameters (such as depth of tumor invasion and lymph node 
metastasis) from case-control studies to predict the clinical outcome of GC. The results demonstrated that telomerase overexpression may play a key role in metastatic progression of GC.

\section{Materials and methods}

Literature search. This meta-analysis followed the proposal set by the Meta-analysis Of Observational Studies in Epidemiology (MOOSE) group (9), and was performed by searching PubMed, MEDLINE, EMBASE, Web of Science databases, Cochrane Library and the Chinese Biomedical Literature database (CBM) (last search updated in October 2011). The search strategy included the following terms: (telomerase [MeSH] or Telomerase Catalytic Subunit [TEXT WORD] or Telomerase Reverse Transcriptase [TEXT WORD] or hTERT [TEXT WORD]) and (Stomach Neoplasms [MeSH] or Gastric Cancer [TEXT WORD] or Gastric Neoplasms [TEXT WORD] or Stomach Cancer [TEXT WORD]). Searches also included scanning reference lists in relevant articles and conference proceedings as well as correspondence with authors when additional data were required. Two reviewers (Lü and Deng) independently screened titles and abstracts of each identified reference, and categorized papers based on the full text to evaluate their eligibility for inclusion.

Inclusion criteria. The inclusion criteria for primary studies were as follows: i) the data were from prospective or retrospective case-control studies and included correlations of telomerase or hTERT to GC; ii) each study presented a proven diagnosis of GC in humans; iii) each study measured telomerase activity or hTERT evaluation using immunohistochemistry (IHC), a telomeric repeat amplification protocol assay (TRAP), a telomeric repeat amplification protocol/enzyme-linked immunosorbent assay (TRAP-ELISA), a membrane-array assay, a reverse transcription-polymerase chain reaction (RT-PCR) or realtime fluorescent quantitative PCR (qRT-PCR); iv) the papers had to provide the sample size, ethnicity and other sample information; v) if data were shared between multiple studies, only the most recent or largest population was included (10), and vi) the publication was in English.

Data extraction. The following items were collected from the reports: first author, year of publication, sample size, ethnicity, TA or hTERT assessment method, cutoff value of TA or hTERT positivity, and telomerase or hTERT expression related to clinicopathological parameters, including gender, age, tumor size, histologic grade, depth of invasion, lymph node metastasis, distant metastasis and TNM stage. Depth of tumor invasion was confirmed using histologic examination, and infiltration into serosa indicated a poor prognosis. The presence of lymph node metastasis in early GC was not a good sign. Distant metastasis was a definite prognostic marker of tumor recurrence. We required that each study definitively reported at least two of the following criteria: the depth of invasion, the presence of lymph node metastasis and the presence of distant metastasis. Data extraction was performed independently by two individuals (Lü and Deng), and any disagreement was resolved by consensus or by consultation with additional reviewers (Yang and Zhang).
Table I. Newcastle-Ottawa quality assessment scale.

Selection

1) Is the case definition adequate?

a) Yes, with independent validation"

b) Yes (record linkage or based on self reports)

c) No description

2) Representativeness of the cases

a) Consecutive or obviously representative series of cases*

b) Potential for selection biases or not stated

3) Selection of controls

a) Community controls*

b) Hospital controls

c) No description

4) Definition of controls

a) No history of disease"

b) No description of source

Comparability

1) Comparability of cases and controls on the basis of the design or analysis

a) Study controls for metastasis

b) Study controls for any additional factor ${ }^{*}$ (age, gender, grade)

Exposure

1) Ascertainment of exposure

a) Secure record (surgical records)

b) Structured interview blind to case/control status*

c) Interview not blinded to case/control status

d) Written self report or medical record only

e) No description

2) Same method of ascertainment for cases and controls

a) Yes*

b) No

3) Non-response rate

a) Same rate for both groups*

b) Non-respondents described

c) Rate different and no designation

A study can be awarded a maximum of one star for each numbered item within the Selection and Exposure categories. A maximum of two stars can be given for Comparability. Underlined and quoted phrases are provided in the scale to allow for adjustment to particular studies. Italicized phrases indicate our interpretation of the question relevant to this study.

Qualitative assessment. Quality assessment was performed with the Newcastle-Ottawa quality assessment scale (NOS) for case-control studies (Table I). A 'star system' has been used to judge data quality based on three broad perspectives: the selection, comparability and outcome of interest for cohort studies. Stars are added up to compare the study quality in a quantitative fashion (11). Based on these criteria, the content validity was evaluated by Lü and Deng, and any disagreement was resolved via discussions between Lü and Deng or with the other authors (Yang and Zhang) for adjudication.

Statistical analysis. Statistical analysis was performed using RevMan 5.0 according to the principles set out in the Cochrane Handbook for Systematic Reviews of Interventions. The methodological quality of each study was assessed with the QUADAS tool recommended by the Cochrane Collaboration, and the kappa statistic $(\kappa)$ for inter-rater reliability was 
calculated. Agreement was assessed using the $\kappa$ statistic for evaluating methodological quality (12). For dichotomous outcomes, the meta-analysis was performed using crude odds ratios (ORs) with $95 \%$ confidence intervals (CIs) to assess the strength of association between telomerase activity or hTERT and metastasis of GC. The data were reported in a binary manner, elucidating the telomerase activity or hTERT value as either 'high' or 'low'. The pooled ORs were conducted to assess the depth of invasion, lymph node metastasis and distant metastasis. For analyzing clinical outcome, well and moderate differentiation were merged, poor and undifferentiated were merged, T1 and T2 were merged, T3 and T4 were merged, stage I and stage II were merged, and stage III and stage IV were merged. Assessment of heterogeneity was assessed by the Chi-square test $\left(\chi^{2}\right)$ and inconsistency index test $\left(\mathrm{I}^{2}\right)$. Heterogeneity was not considered statistically significant when $p>0.10$ in the $\chi^{2}$-test, and acceptable heterogeneity was defined as $\mathrm{I}^{2}<50 \%$ in studies. For studies lacking a measure of heterogeneity, a Mantel-Haenszel fixed effect model was used for the primary meta-analysis (13); otherwise, a DerSimonianLaird random effects model was adopted (14). Assessment of publication bias for each of the pooled study groups was tested using a funnel plot.

\section{Results}

Selection and characteristics of the studies. At the beginning, 215 records were examined according to the search strategies. In total, 152 articles were eliminated after scanning the titles or abstracts since they were review articles, case reports, commentaries and letters or since they were irrelevant to this analysis. After further review, an additional 45 articles were excluded: first, 2 studies overlapped with others. Second, 9 studies were experiments on cell cultures or animals. Finally, 34 studies lacked usable data that correlated telomerase or hTERT with lymph node status or TNM stage to create $2 \times 2$ tables. Thus, a total of 18 eligible studies related to GC patients were finally identified in our meta-analysis with good agreement between reviewers ( $\kappa=0.73)(15-32)$ (Fig. 1).

In the remaining studies, all measurements were performed using the primary tumor, and all of the patients had not received chemotherapy or radiotherapy before enrollment. Although research was conducted at tertiary referral centers, almost all studies were performed in Asia and one in South America (22). Sample size varied from 20 to 95 participants, and the average age across all of the studies was 59.3 years, with a variation ranging from 32 to 89 years. The number of GC patients with T3 and T4 invasion ranged from 11 to 78 ; the number of GC patients with lymph node metastasis ranged from 7 to 75 ; the number of GC patients with distant metastasis ranged from 2 to 32 . Four studies used IHC, 9 studies used TRAP, 1 study used TRAPELISA, 1 study used a membrane-array assay, 2 studies used RT-PCR and 1 study used qRT-PCR. The quality assessment of studies was performed using the NOS ranged from 5 to 7 (with a mean star rating of 5.9), with a higher value indicating better methodology. The scale is listed in Table II. The cutoff value of telomerase or hTERT expression was determined using different methods in each study. The basic feature description of the 18 studies is summarized in Table II, and

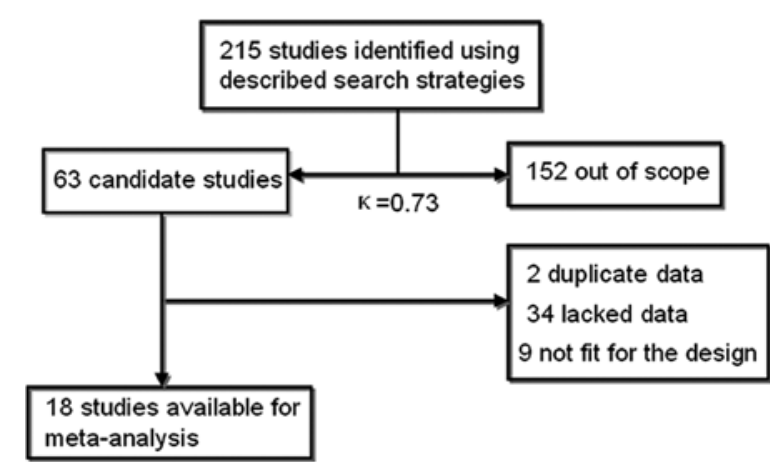

Figure 1. Flow chart of the meta-analysis.

the correlation between telomerase or hTERT expression and clinicopathological factors is listed in Table III.

\section{Quantitative synthesis}

Correlation between TA and clinicopathological characteristics. When stratifying variables by lymph node metastasis, there was a slight heterogeneity in the data $\left(\chi^{2}=26\right.$, $\left.\mathrm{I}^{2}=38.5 \%, \mathrm{p}=0.05\right)$. Patients with lymph node metastasis in GC displayed a significantly higher TA expression in 17 studies (866 patients) (OR=2.03, 95\% CI 1.21-3.39, $\mathrm{p}=0.007$; Fig. 2A). When stratifying for the depth of tumor invasion in GC, 18 studies (886 patients) were reported without significant heterogeneity $\left(\chi^{2}=21.34, \mathrm{I}^{2}=20.3 \%, \mathrm{p}=0.21\right)$. We observed that patients with T3 and T4 GC had a significantly higher TA (OR=1.87, 95\% CI 1.30-2.70, p=0.0007; Fig. 2B). When stratifying for distant metastasis in GC, 9 studies (407 patients) were combined, and there was no significant heterogeneity in the data $\left(\chi^{2}=11.95, \mathrm{I}^{2}=33 \%, \mathrm{p}=0.15\right)$. Patients with distant metastasis had a significantly higher TA in GC (OR=2.71, 95\% CI 1.59-4.63, p=0.0002; Fig. 2C). We also observed a correlation between TA and other clinical characteristics, including tumor size $>5 \mathrm{~cm}$ in 9 studies (466 patients; OR=2.14, 95\% CI 1.31-3.50, $\mathrm{p}=0.002$; Fig. 2D), poor histologic differentiation in 16 studies (791 patients; $\mathrm{OR}=1.51,95 \%$ CI $0.73-3.11, \mathrm{p}=0.26$; Fig. 2E), and a higher (III + IV) clinical stage in 14 studies (711 patients; $\mathrm{OR}=2.39$, 95\% CI 1.30-4.41, $\mathrm{p}=0.005$; Fig. 2F). When stratifying the variables by poor histologic differentiation of GC, there was heterogeneity $\left(\mathrm{I}^{2}=60.4 \%\right)$, then the DerSimonian-Laird random effects model was used. There was no significant correlation between TA and histologic differentiation $(\mathrm{p}=0.26)$.

Publication bias. Funnel plots were used to estimate the publication bias of the meta-analysis. As shown in Fig. 3, the shape of the funnel plot did not reveal obvious asymmetry.

\section{Discussion}

GC remains the second leading cause of cancer-related mortality worldwide (33), in part because of its high rate of metastasis and recurrence. It is critical to explore molecular biomarkers to guide clinical decision-making with regard to the treatment of GC. TA is thought to be a critical step in the evolution of most tumor types $(34-37)$, including GC $(38,39)$. Considerable clinical research has been conducted with the aim of assessing the correlation between TA expression and clinicopathological outcome in patients with GC, but the 
Table II. Main characteristics of the 18 studies included in the meta-analysis.

\begin{tabular}{|c|c|c|c|c|c|c|c|c|c|c|}
\hline Author/(Ref.) & $\begin{array}{c}\text { Year of } \\
\text { publication }\end{array}$ & Language & Population & $\begin{array}{c}\text { Study } \\
\text { from } \\
\text { PubMed }\end{array}$ & $\begin{array}{l}\text { No. of } \\
\text { patients } \\
(\mathrm{M} / \mathrm{F})\end{array}$ & $\begin{array}{l}\text { Median } \\
\text { age } \\
\text { (years) }\end{array}$ & $\begin{array}{l}\text { TA/hTERT } \\
\text { detection } \\
\text { method }\end{array}$ & $\begin{array}{l}\text { Cutoff for TA } \\
\text { positivity } \\
(\%)\end{array}$ & Result & $\begin{array}{l}\text { Study } \\
\text { quality } \\
\text { points }\end{array}$ \\
\hline Yang et al (15) & 2001 & English & China & Yes & $29 / 13$ & 52.9 & TRAP assay & $>6$-bp ladder & All negative & $7 / 9$ \\
\hline Liu et al (27) & 2008 & Chinese & China & Yes & $27 / 13$ & 54.0 & RT-PCR & $>0.6$ & $3,4,6$ positive & $6 / 9$ \\
\hline Okusa et al (28) & 1998 & English & Japan & Yes & $22 / 14$ & 62.3 & TRAP-ELISA & $>5 \%$ & 5 positive & $6 / 9$ \\
\hline Hu et al (16) & 2009 & English & China & Yes & $28 / 18$ & 56.3 & TRAP assay & $>0.2$ units & All positive & $5 / 9$ \\
\hline Shin et al (29) & 2002 & English & Korea & Yes & $35 / 30$ & 55.4 & RT-PCR & NR & 2,4 positive & $6 / 9$ \\
\hline Mori et al (30) & 2000 & English & Japan & Yes & $32 / 14$ & 61.7 & TRAP assay & $>6$-bp ladder & 3 positive & $5 / 9$ \\
\hline Wu et al (17) & 2006 & English & China & Yes & $41 / 23$ & 60.5 & $\begin{array}{l}\text { Membrane- } \\
\text { array assay }\end{array}$ & ROC curve & All negative & $7 / 9$ \\
\hline Wang et al (18) & 2004 & English & China & Yes & $30 / 11$ & 57.2 & IHC & $>5 \%$ & $1,2,4,6$ positive & $6 / 9$ \\
\hline Yoo et al (19) & 2003 & English & Korea & Yes & $38 / 13$ & 61.3 & IHC & $>10 \%$ & 2,3 positive & $6 / 9$ \\
\hline Yasui et al (20) & 1998 & English & Japan & Yes & $10 / 10$ & 68.9 & IHC & $\begin{array}{l}\text { Focal or } \\
\text { diffuse staining }\end{array}$ & NR & $5 / 9$ \\
\hline $\begin{array}{l}\text { Kameshima } \\
\text { et al (21) }\end{array}$ & 2000 & English & Japan & Yes & $19 / 8$ & 66.9 & TRAP assay & $>0.6 \mu \mathrm{g}$ & All negative & $6 / 9$ \\
\hline Gigek et al (22) & 2009 & English & Brasil & Yes & $36 / 19$ & NR & $\mathrm{IHC}$ & $\begin{array}{l}\text { No positive } \\
\text { cells were } \\
\text { observed }\end{array}$ & All negative & $5 / 9$ \\
\hline Hu et al (23) & 2004 & English & China & Yes & $25 / 10$ & 55.2 & qRT-PCR & $>5.39$ & 2 positive & $6 / 9$ \\
\hline Ahn et al (24) & 1997 & English & Korea & Yes & $57 / 38$ & 54.3 & TRAP assay & $>6$-bp ladder & All negative & $7 / 9$ \\
\hline Zhan et al (31) & 1999 & English & China & Yes & $50 / 44$ & 63.0 & TRAP assay & $>6$-bp ladder & All negative & $6 / 9$ \\
\hline $\begin{array}{l}\text { Gümüx-Akay } \\
\text { et al (32) }\end{array}$ & 2007 & English & Turkey & Yes & NR & NR & TRAP assay & NR & $2,3,4$ negative & $5 / 9$ \\
\hline Hiyama et al (25) & 1995 & English & Japan & Yes & $37 / 19$ & 55.0 & TRAP assay & $>0.6 \mu \mathrm{g}$ & 1,4,6 positive & $7 / 9$ \\
\hline Tahara et al (26) & 1995 & English & Japan & Yes & $13 / 7$ & 64.0 & TRAP assay & $>6$-bp ladder & 4,5 positive & $6 / 9$ \\
\hline
\end{tabular}

Results: 1 , tumor size; 2 , histologic grade; 3 , depth of invasion; 4, lymph node metastasis; 5 , distant metastasis; 6 , TNM stage. Study quality is listed using the results of the Newcastle-Ottawa questionnaire. NR, not reported.

Table III. Main characteristics of 18 studies relating TA expression to clinicopathological factors.

\begin{tabular}{|c|c|c|c|c|c|c|c|c|c|c|}
\hline \multirow[t]{2}{*}{ Author/(Ref.) } & \multirow{2}{*}{$\begin{array}{l}\text { Year of } \\
\text { publication }\end{array}$} & \multirow[t]{2}{*}{ Language } & \multirow[t]{2}{*}{ Country } & \multirow{2}{*}{$\begin{array}{l}\text { No. of positive/ } \\
\text { (negative) }\end{array}$} & \multicolumn{6}{|c|}{ No. of patients with TA-positivity } \\
\hline & & & & & $\begin{array}{c}\text { Size } \\
>5 \mathrm{~cm} \\
(<5 \mathrm{~cm})\end{array}$ & $\begin{array}{c}\text { Histo } \\
\text { P/U } \\
(\mathrm{W} / \mathrm{M})\end{array}$ & $\begin{array}{c}\mathrm{T} \\
\mathrm{T} 3 / \mathrm{T} 4 \\
(\mathrm{~T} 1 / \mathrm{T} 2)\end{array}$ & $\begin{array}{c}\mathrm{N} \\
\text { positive/ } \\
\text { (negative) }\end{array}$ & $\begin{array}{c}\mathrm{M} \\
\text { positive/ } \\
\text { (negative) }\end{array}$ & $\begin{array}{c}\text { TNM } \\
\text { TIII/IV } \\
\text { (TI/TII) }\end{array}$ \\
\hline Liu et al (27) & 2008 & English & China & $26(14)$ & - & - & $20(6)$ & $22(4)$ & $11(15)$ & $24(2)$ \\
\hline Yang et al (15) & 2001 & English & China & $40 \quad(2)$ & $26(14)$ & $30(10)$ & $26(14)$ & $20(20)$ & - & $17(23)$ \\
\hline Wu et al (17) & 2006 & English & China & $52(12)$ & $23(29)$ & $49 \quad(3)$ & $40(12)$ & $37(15)$ & $14(38)$ & $33(19)$ \\
\hline Hu et al (23) & 2004 & English & China & $18(17)$ & 10 & 14 (4) & $7(11)$ & 14 (4) & $10(8)$ & - \\
\hline Zhan et al (31) & 1999 & English & China & $81(13)$ & $46(35)$ & $43(38)$ & $68(13)$ & $51(30)$ & - & $60(21)$ \\
\hline Hu et al (16) & 2009 & English & China & $41 \quad(5)$ & $28(13)$ & 33 & $21(20)$ & $5(36)$ & $30(11)$ & - \\
\hline Wang et al (18) & 2004 & English & China & $32(9)$ & $19(13)$ & 25 & 13 (19) & $23(9)$ & - & $27 \quad(5)$ \\
\hline Gümüx-Akay et al (32) & 2007 & English & China & 42 (1) & - & $22(20)$ & $31(11)$ & $27(15)$ & - & - \\
\hline Ahn et al (24) & 1997 & English & Korea & $85(10)$ & - & $63(22)$ & $60(25)$ & $68(17)$ & - & $61(24)$ \\
\hline Yoo et al (19) & 2003 & English & Korea & $37(14)$ & 18 (19) & $20(17)$ & $11(26)$ & $26(11)$ & - & - \\
\hline Shin et al (29) & 2002 & English & Korea & $30(35)$ & - & $20(10)$ & $10(20)$ & $22(8)$ & $7(23)$ & $13(17)$ \\
\hline Mori et al (30) & 2000 & English & Japan & $19(27)$ & - & 10 & $16(3)$ & $14 \quad(5)$ & $6(13)$ & 12 (7) \\
\hline Tahara et al (26) & 1995 & English & Japan & 17 (3) & - & 8 (9) & 14 (3) & 12 (5) & $2(15)$ & $12(5)$ \\
\hline Hiyama et al (25) & 1995 & English & Japan & $56(10)$ & $28(28)$ & 51 & $42(14)$ & $34(22)$ & - & $24(32)$ \\
\hline Okusa et al (28) & 1998 & English & Japan & $22(14)$ & - & $10(12)$ & 20 & 18 (4) & $8(14)$ & $16(6)$ \\
\hline Yasui et al (20) & 1998 & English & Japan & 17 (3) & - & $9(8)$ & $16(1)$ & - & - & $11(6)$ \\
\hline Kameshima et al (21) & 2000 & English & Japan & $19(8)$ & $8(11)$ & $7(12)$ & 11 & $8(11)$ & - & $7(12)$ \\
\hline Gigek et al (22) & 2009 & English & Brasil & $44(11)$ & - & - & $23(21)$ & $43 \quad(1)$ & $18(26)$ & 43 (1) \\
\hline
\end{tabular}

Positive, patient have TA expression; negative, patients have no TA expression. T, depth of invasion. N, lymph node metastasis. M, distant metastasis. Histo, histodifferentiation: P, poor differentiation; U, undifferentiation; W, well differentiation; M, moderate differentiation; '-' corresponds to missing data and was not analyzed in the meta-analysis. 

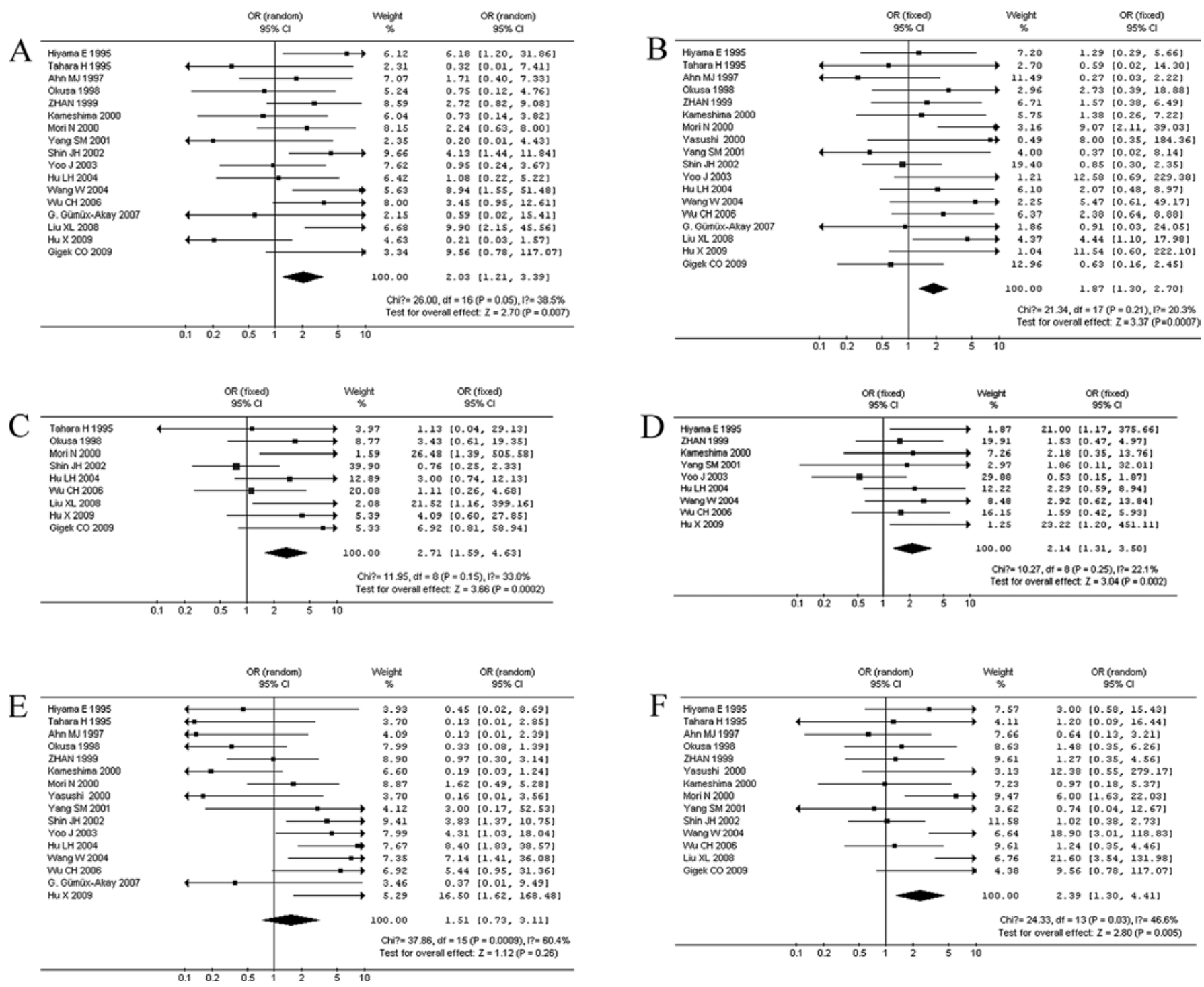

Figure 2. Meta-analysis of the relation between TA expression and clinicopathological parameters. (A) Lymph node metastasis, (B) depth of tumor invasion, (C) distant metastasis, (D) tumor size, (E) histologic differentiation and (F) clinical stage.

results have been controversial. Several studies have shown that the TA in GC tissues is related to depth of tumor invasion and lymph node metastasis $(25,29)$; however, other studies found no association between clinical outcome and TA $(21,24)$.

Recently, many studies have indicated that hTERT is the rate-limiting step in the activation of telomerase, and its expression level is directly proportional to expression levels of TA $(40,41)$. In our analysis, TA was measured by TRAP assay in 9 studies and TRAP-ELISA in 1 study, whereas hTERT was measured by RT-PCR in 2 studies, qRT-PCR in 1 study, a membrane-array assay in 1 study and IHC in 4 studies. TA expression was detected using all of these methods. The cutoff value of TA positivity obtained from different methods was recognized as a standard to assess TA expression. The pooled statistical data showed that the prognostic utility of TA was consistent with clinical characteristics, including depth of tumor invasion $(\mathrm{p}=0.0007)$, lymph node status $(\mathrm{p}=0.007)$, distant metastasis $(\mathrm{p}=0.0002)$ and TNM stage $(\mathrm{p}=0.005)$. In addition, we identified and evaluated the association of TA expression with tumor size and tumor grade. Our findings also showed that there was a strong association between high TA expression potential and tumor size $(\mathrm{p}=0.002)$, but not tumor grade $(p=0.26)$. Combining several independent studies, our estimates supported the idea that TA and hTERT overexpression were strongly related to gastric tumor invasion and metastasis. Therefore, the role telomerase plays in inducing tumor progression is not only based on its well-documented effects on tumor proliferation rate (42).

Our findings were consistent with the reports on TA expression in melanoma (43), breast cancer (44), hepatocellular carcinoma (45) and giant-cell tumors of the bone (7), in which TA contributed to the poor survival of patients. We demonstrated at the cellular level that hTERT transfection in U2OS (a hTERT-negative cell line) re-activated its telomerase activity and further promoted its invasive and metastatic potential. The mechanism that enhances these malignant phenotypes may be correlated with the increasing adhesive ability of these tumor cells to the extracellular matrix (46). High TA may activate the glycolytic pathway to promote tumor growth and metastasis (43). TA suppression may render cells more susceptible to anchorage-independent growth inhibition, and unstable tumors require a higher TA level to prevent genomic deterioration and 

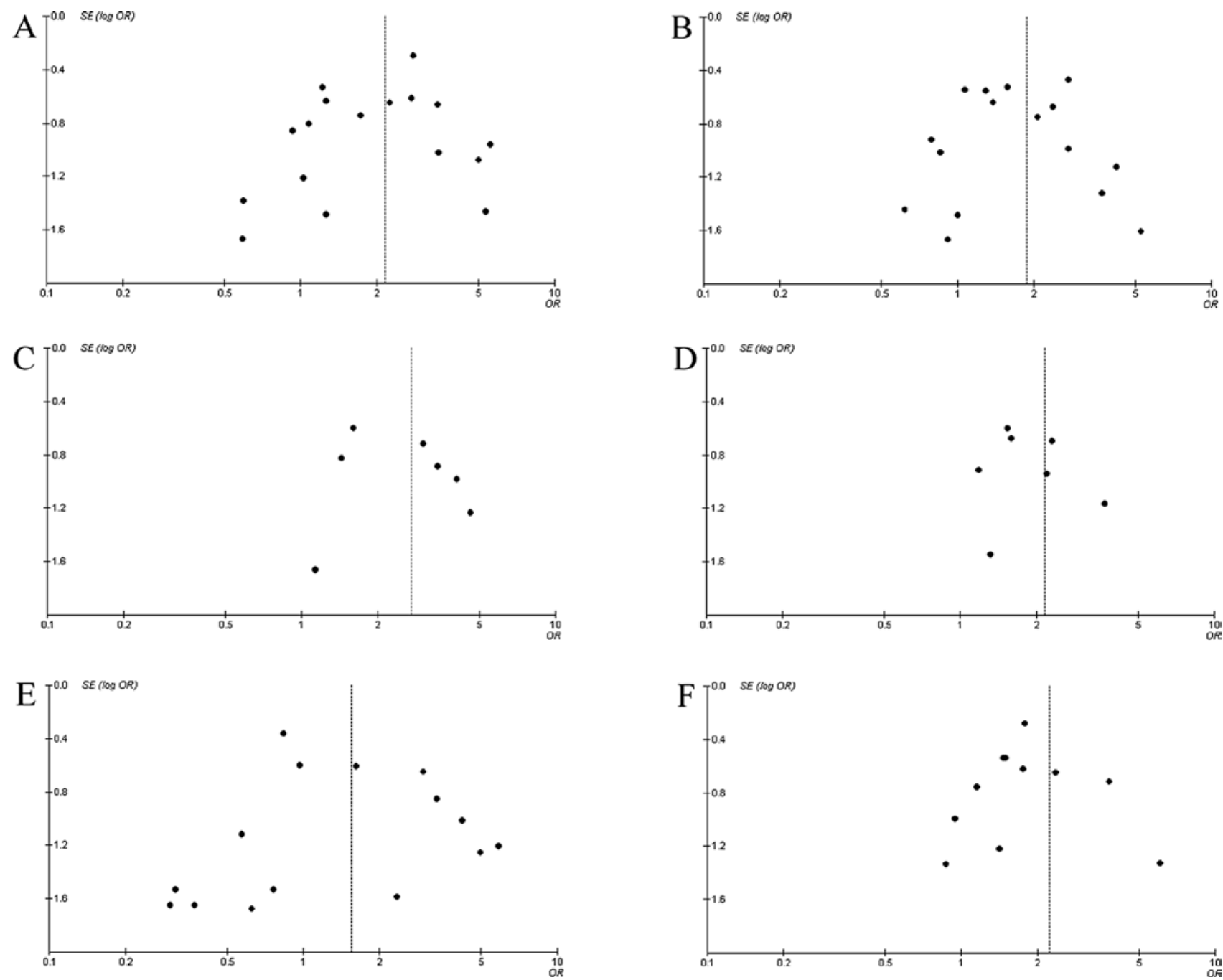

Figure 3. Funnel plot analysis of publication bias for clinicopathological parameters. (A) Lymph node metastasis, (B) depth of tumor invasion, (C) distant metastasis, (D) tumor size, (E) histologic differentiation and (F) clinical stage.

to induce more aggressive cancer cells during carcinogenesis (7). The mechanism described above offered a possible interpretation of the observed strong statistical association between TA overexpression and tumor metastasis.

Caution must be taken to note the limitations of this study. First, most of the patients with GC enrolled in our meta-analysis came from Asia, which may be attributed to the apparent decrease in the incidence and mortality rates for GC in the past 50 years in Western countries compared to Japan and China (47). Second, reports in languages other than English were excluded. The risk of language bias had to be considered, but may not result in any notable bias in the assessment of interventional effectiveness (48). Third, data containing negative results may be less likely published, although we took care to access all available data. Fourth, the eligible data do not assess whether TA may influence the prognosis of patients according to distinct therapeutic schedules.

Based on the results of this analysis, we conclude that telomerase overexpression is not only involved in the carcinogenesis in the initiation of $\mathrm{GC}$, but also promotes the invasion and metastasis of GC. These results improve our understanding of telomerase as a potentially important molecular target in clinical diagnostics and therapeutics of gastric cancer.

\section{Acknowledgements}

The authors would like to thank all of the patients and clinical investigators who were involved in the studies selected for this meta-analysis.

\section{References}

1. Roder DM: The epidemiology of gastric cancer. Gastric Cancer 5 (Suppl 1): 5-11, 2002.

2. Yokota J: Tumor progression and metastasis. Carcinogenesis 21: 497-503, 2000.

3. Greider CW and Blackburn EH: A telomeric sequence in the RNA of Tetrahymena telomerase required for telomere repeat synthesis. Nature 337: 331-337, 1989.

4. Sealey DC, Zheng L, Taboski MA, Cruickshank J, Ikura M and Harrington LA: The N-terminus of hTERT contains a DNA-binding domain and is required for telomerase activity and cellular immortalization. Nucleic Acids Res 38: 2019-2035, 2010.

5. Catarino R, Araujo A, Coelho A, Gomes M, Nogueira A, Lopes C and Medeiros RM: Prognostic significance of telomerase polymorphism in non-small cell lung cancer. Clin Cancer Res 16: 3706-3712, 2010.

6. Poremba C, Heine B, Diallo R, Heinecke A, Wai D, Schaefer KL, Braun Y, Schuck A, Lanvers C, Bankfalvi A, et al: Telomerase as a prognostic marker in breast cancer: high-throughput tissue microarray analysis of hTERT and hTR. J Pathol 198: 181-189, 2002. 
7. Horvai AE, Kramer MJ, Garcia JJ and O'Donnell RJ: Distribution and prognostic significance of human telomerase reverse transcriptase (hTERT) expression in giant-cell tumor of bone. Mod Pathol 21: 423-430, 2008

8. Stroup DF, Berlin JA, Morton SC, Olkin I, Williamson GD, Rennie D, Moher D, Becker BJ, Sipe TA and Thacker SB: Metaanalysis of observational studies in epidemiology: a proposal for reporting. Meta-analysis Of Observational Studies in Epidemiology (MOOSE) group. JAMA 283: 2008-2012, 2000.

9. Little J, Bradley L, Bray MS, Clyne M, Dorman J, Ellsworth DL, Hanson J, Khoury M, Lau J, O'Brien TR, et al: Reporting, appraising, and integrating data on genotype prevalence and gene-disease associations. Am J Epidemiol 156: 300-310, 2002.

10. Stang A: Critical evaluation of the Newcastle-Ottawa scale for the assessment of the quality of nonrandomized studies in metaanalyses. Eur J Epidemiol 25: 603-605, 2010.

11. Whiting PF, Weswood ME, Rutjes AW, Reitsma JB, Bossuyt PN and Kleijnen J: Evaluation of QUADAS, a tool for the quality assessment of diagnostic accuracy studies. BMC Med Res Methodol 6: 9, 2006.

12. Cohen J: A coefficient of agreement for nominal scales. Educ Psychol Meas 20: 37-46, 1960.

13. Greenland S and Robins J: Estimation of a common effect parameter from sparse follow-up data. Biometrics 41: 55-68, 1990.

14. DerSimonian R and Laird N: Meta-analysis in clinical trials. Control Clin Trials 7: 177-188, 1986.

15. Yang SM, Fang DC, Luo YH, Lu R, Battle PD and Liu WW: Alterations of telomerase activity and terminal restriction fragment in gastric cancer and its premalignant lesions. J Gastroenterol Hepatol 16: 876-882, 2001.

16. $\mathrm{Hu} \mathrm{X}, \mathrm{Wu} \mathrm{H}$, Zhang S, Yuan $\mathrm{H}$ and Cao L: Clinical significance of telomerase activity in gastric carcinoma and peritoneal dissemination. J Int Med Res 37: 1127-1138, 2009.

17. Wu CH, Lin SR, Yu FJ, Wu DC, Pan YS, Hsieh JS, Huang SY and Wang JY: Development of a high-throughput membrane-array method for molecular diagnosis of circulating tumor cells in patients with gastric cancers. Int J Cancer 119: 373-379, 2006.

18. Wang W, Luo HS and Yu BP: Expression of NF-kappaB and human telomerase reverse transcriptase in gastric cancer and precancerous lesions. World J Gastroenterol 10: 177-181, 2004.

19. Yoo J, Park SY, Kang SJ, Kim BK, Shim SI and Kang CS Expression of telomerase activity, human telomerase RNA, and telomerase reverse transcriptase in gastric adenocarcinomas. Mod Pathol 20: 700-707, 2003

20. Yasui W, Tahara H, Tahara E, Fujimoto J, Nakayama J, Ishikawa F and Ide T: Expression of telomerase catalytic component, telomerase reverse transcriptase, in human gastric carcinomas. Jpn J Cancer Res 89: 1099-1103, 1998.

21. Kameshima H, Yagihashi A, Yajima T, Kobayashi D, Denno R, Hirata $\mathrm{K}$ and Watanabe $\mathrm{N}$ : Helicobacter pylori infection: augmentation of telomerase activity in cancer and noncancerous tissues. World J Surg 24: 1243-1249, 2000.

22. Gigek CO, Leal MF, Silva PN, Lisboa LC, Lima EM, Calcagno DQ, Assumpcao PP, Burbano RR and Smith Mde A: hTERT methylation and expression in gastric cancer. Biomarkers 14: 630-636, 2009.

23. Hu LH, Chen FH, Li YR and Wang L: Real-time determination of human telomerase reverse transcriptase mRNA in gastric cancer. World J Gastroenterol 10: 3514-3517, 2004

24. Ahn MJ, Noh YH, Lee YS, Lee JH, Chung TJ, Kim IS, Choi IY, Kim SH, Lee JS and Lee KH: Telomerase activity and its clinicopathological significance in gastric cancer. Eur J Cancer 33 1309-1313, 1997

25. Hiyama E, Yokoyama T, Tatsumoto N, Hiyama K, Imamura Y, Murakami Y, Kodama T, Piatyszek MA, Shay JW and Matsuura Y: Telomerase activity in gastric cancer. Cancer Res 55: 3258-3262, 1995

26. Tahara H, Kuniyasu H, Yokozaki H, Yasui W, Shay JW, Ide T and Tahara E: Telomerase activity in preneoplastic and neoplastic gastric and colorectal lesions. Clin Cancer Res 1: 1245-1251, 1995.

27. Liu X1, Chen P and Wei JM: The relationship between CK20 mRNA and hTERT mRNA expression in peripheral blood of patients with gastric carcinoma and tumor micrometastasis. Chin J Clin Oncol 35: 1286-1289, 2008.

28. Okusa Y, Shinomiyo N, Ichikura T and Mochizuki H: Correlation between telomerase activity and DNA ploidy in gastric cancer. Oncology 55: 258-264, 1998.

29. Shin JH, Chung J, Kim HO, Kim YH, Hur YM, Rhim JH, Chung HK, Park SC, Park JG and Yang HK: Detection of cancer cells in peripheral blood of stomach cancer patients using RT-PCR amplification of tumour-specific mRNAs. Aliment Pharmacol Ther 16 (Suppl 2): 137-144, 2002.
30. Mori N, Oka M, Hazama S, Iizuka N, Yamamoto K, Yoshino S, Tangoku A, Noma T and Hirose K: Detection of telomerase activity in peritoneal lavage fluid from patients with gastric cancer using immunomagnetic beads. Br J Cancer 83: 1026-1032, 2000.

31. Zhan WH, Ma JP, Peng JS, Gao JS, Cai SR, Wang JP, Zheng ZQ and Wang L: Telomerase activity in gastric cancer and its clinical implications. World J Gastroenterol 5: 316-319, 1999.

32. Gümüx-Akay G, Ünal AE, Bayar S, Karadayi K, Elhan AH, Sunguroqlu A and Tükün A: Telomerase activity could be used as a marker for neoplastic transformation in gastric adenocarcinoma: but it does not have a prognostic significance. Genet Mol Res 6: 41-49, 2007 (In Bulgarian).

33. Bulanov D: Gastric Cancer - Current state of the problem. Part I. Epidemiology. Pathology. Classification. Staging. Khirurgiia (Sofia), 48-59, 2007 (In Bulgarian).

34. Beisner J, Dong M, Taetz S, Nafee N, Griese EU, Schaefer U, Lehr CM, Klotz U and Murdter TE: Nanoparticle mediated delivery of 2'-O-methyl-RNA leads to efficient telomerase inhibition and telomere shortening in human lung cancer cells. Lung Cancer 68: 346-354, 2010.

35. Lu L, Zhang C, Zhu G, Irwin M, Risch H, Menato G, Mitidieri M, Katsaros D and $\mathrm{Yu} \mathrm{H}$ : Telomerase expression and telomere length in breast cancer and their associations with adjuvant treatment and disease outcome. Breast Cancer Res 13: R56, 2011.

36. Nakamura M, Saito H, Ebinuma H, Wakabayashi K, Saito Y, Takagi T, Nakamoto $\mathrm{N}$ and Ishii H: Reduction of telomerase activity in human liver cancer cells by a histone deacetylase inhibitor. J Cell Physiol 187: 392-401, 2001.

37. Capezzone M, Cantara S, Marchisotta S, Filetti S, De Santi MM, Rossi B, Ronga G, Durante C and Pacini F: Short telomeres, telomerase reverse transcriptase gene amplification, and increased telomerase activity in the blood of familial papillary thyroid cancer patients. J Clin Endocrinol Metab 93: 3950-3957, 2008.

38. Miyachi K, Fujita M, Tanaka N, Sasaki K and Sunagawa M: Correlation between telomerase activity and telomeric-repeat binding factors in gastric cancer. J Exp Clin Cancer Res 21: $269-275,2002$

39. Gumus-Akay G, Elhan AH, Unal AE, Demirkazik A, Sunguroglu A and Tukun A: Effects of genomic imbalances on telomerase activity in gastric cancer: clues to telomerase regulation. Oncol Res 17: 455-462, 2009.

40. Usselmann B, Newbold M, Morris AG and Nwokolo CU: Telomerase activity and patient survival after surgery for gastric and oesophageal cancer. Eur J Gastroenterol Hepatol 13: 903-908, 2001

41. Poole JC, Andrews LG and Tollefsbol TO: Activity, function, and gene regulation of the catalytic subunit of telomerase (hTERT). Gene 269: 1-12, 2001

42. Jin X, Beck S, Sohn YW, Kim JK, Kim SH, Yin J, Pian X, Kim SC, Choi YJ and Kim H: Human telomerase catalytic subunit (hTERT) suppresses p53-mediated anti-apoptotic response via induction of basic fibroblast growth factor. Exp Mol Med 42: 574-582, 2010.

43. Bagheri S, Nosrati M, Li S, Fong S, Torabian S, Rangel J, Moore DH, Federman S, Laposa RR, Baehner FL, et al: Genes and pathways downstream of telomerase in melanoma metastasis. Proc Natl Acad Sci USA 103: 11306-11311, 2006.

44. Hochreiter AE, Xiao H, Goldblatt EM, Gryaznov SM, Miller KD, Badve S, Sledge GW and Herbert BS: Telomerase template antagonist GRN163L disrupts telomere maintenance, tumor growth, and metastasis of breast cancer. Clin Cancer Res 12: 3184-3192, 2006.

45. Oh BK, Kim H, Park YN, Yoo JE, Choi J, Kim KS, Lee JJ and Park C: High telomerase activity and long telomeres in advanced hepatocellular carcinomas with poor prognosis. Lab Invest 88: 144-152, 2008

46. Yu ST, Chen L, Wang HJ, Tang XD, Fang DC and Yang SM: hTERT promotes the invasion of telomerase-negative tumor cells in vitro. Int J Oncol 35: 329-336, 2009.

47. Palli D: Epidemiology of gastric cancer. Ann Ist Super Sanita 32: 85-99, 1996.

48. Soler RE, Leeks KD, Razi S, Hopkins DP, Griffith M, Aten A, Chattopadhyay SK, Smith SC, Habarta N, Goetzel RZ, et al: A systematic review of selected interventions for worksite health promotion. The assessment of health risks with feedback. Am J Prev Med 38: S237-S262, 2010. 